\title{
Aortic stenosis concomitant with microscopic polyangiitis: a challenge in medical reasoning and thinking
}

\author{
Paulo Sampaio Gutierrez ${ }^{a}$, Vera Demarchi Aielloa
}

Gutierrez PS, Aiello VD. Aortic stenosis concomitant with microscopic polyangiitis: a challenge in medical reasoning and thinking. Autopsy Case Rep [Internet]. 2014; 4(1): 7-14. http://dx.doi.org/10.4322/acr.2014.002

\section{ABSTRACT}

Microscopic polyangiitis (MPA) is part of the anti-neutrophil cytoplasmic antibodies (ANCA)-related vasculitis, which usually presents as renal pulmonary syndrome. It is defined as a pauci-immune necrotizing small vessel vasculitis, which usually affects the kidneys, followed by the lungs. It also presents systemic symptoms. The etiology of MPA is still unclear, but evidence reinforces the autoimmune mechanisms as the main etiopathogenic factor. Aortic valve stenosis (AS) is not an uncommon disease whose etiology varies according to geographical differences and the patient's age. The natural history of AS begins with a prolonged asymptomatic period, but when symptomatic, respiratory failure is one of its main clinical presentations. The authors present the case of a 55-year-old woman who was admitted with the diagnosis of renal failure, anemia, and a cardiac murmur. The patient had been recently diagnosed with pneumonia. During hospitalization, diagnostic workup disclosed a normal kidney size as well as parenchymal thickness. A renal biopsy was undertaken but the specimen was exiguous, showing 4 sclerotic glomeruli and 1 glomerulus with crescentic glomerulonephritis. The search for ANCA was positive. The investigation of the cardiac murmur disclosed AS. The patient, on hemodialysis, presented episodes of respiratory failure, which was interpreted as acute pulmonary edema, but a suspicion of ANCA-related pulmonary renal syndrome was raised. However, the aortic valve replacement was prioritized. While awaiting cardiac surgery, the patient died because of respiratory insufficiency. Autopsy findings concluded that MPA with pulmonary hemorrhage due to vasculitis was the immediate cause of death. Although AS was present at autopsy and classified as moderate/severe, this lesion was a bystander in the process of this patient's end of life, demonstrating the value of autopsy for medical learning and reasoning purposes.

Keywords: Systemic Vasculitis; Aortic Valve Stenosis; Renal Insufficiency; Respiratory Insufficiency; Antibodies, Antineutrophil Cytoplasmic; Glomerulonephritis.

\footnotetext{
a Laboratory of Pathology - Instituto do Coração - Hospital das Clínicas - Faculdade de Medicina - Universidade de São Paulo, São Paulo/SP - Brazil.
} 


\section{CASE REPORT}

A 55-year-old female patient sought the medical facility complaining of a 6-day history of diffuse abdominal pain radiating for flanks, accompanied by nausea and chronic constipation. She denied fever and vomiting. She had been using a non-steroid anti-inflammatory drug (NSAID) during the last week because of discomfort in her lower limbs. She referred recent treatment for pneumonia, and progressive exertion dyspnea during the last year, the latter of which did not disable her daily activities. Her past medical history included the diagnosis of a cardiac murmur for at least 11 years without symptoms of congestive heart failure; an appendectomy; a hysterectomy over 20 years ago; and tabagism of 90 packs/year.

The initial physical examination revealed a pale but well-looking patient, afebrile, with normal hemodynamic parameters, and no peripheral edema. Lung examination was normal, and cardiac auscultation revealed an ejection murmur more audible over the aortic area. The abdomen was flat, although slightly tender; the bowel sounds were normal; and neither peritoneal irritation nor Giordano's sign were present. Initial laboratory workup revealed renal failure: creatinine $=7.6$ $\mathrm{mg} / \mathrm{dL}$ (reference value [RV]: $0.4-1.3 \mathrm{mg} / \mathrm{dL}$ ) and urea $=191 \mathrm{mg} / \mathrm{dL}(\mathrm{RV}: 10-50 \mathrm{mg} / \mathrm{dL})$. The blood cell count was normal except for the low hemoglobin determination $=8 \mathrm{~g} / \mathrm{dL}(\mathrm{RV}: 12.3-15.3 \mathrm{~g} / \mathrm{dL})$ and hematocrit $=27 \%$ (RV: $36 \cdot 0-45.0 \%$ ). (Iron profile tests were compatible with iron-deficiency anemia.) Potassium was $5.6 \mathrm{mEq} / \mathrm{L}$ (RV: $3.5-5.0 \mathrm{mEq} / \mathrm{L}$ ) and the remaining electrolytes and $\mathrm{pH}$ were within normal limits. Urinalysis revealed hematuria; 24-hour proteinuria $=689 \mathrm{mg}$. Urinary tract ultrasonography revealed normal kidney size and parenchymal thickening. The echodopplercardiogram showed an enlarged left atrium $(47 \mathrm{~mm})$ and a left ventricular ejection fraction of $62 \%$. $^{\text {b }}$ The septum measured $17 \mathrm{~mm}$ and the posterior wall $14 \mathrm{~mm}$. Inferolateral hypokinesia was present. Acalcified aortic valve with marked stenosis showed a maximum left ventricular aortic gradient pressure of $92 \mathrm{mmHg}$.

The patient was admitted with the hypothesis of non-olyguric acute renal failure, which was initially considered as NSAID-induced acute interstitial nephritis, and severe aortic stenosis (AS). Therefore,

b Pombo JF, Troy BL, Russell RO Jr. Left ventricular volumes and ejection fraction by echocardiography. Circulation. 1971;43:480-90. she was started on prednisone $1 \mathrm{mg} / \mathrm{kg} /$ day, but no improvement of renal function was observed, so the patient went on hemodialysis treatment.

A renal biopsy was undertaken, which revealed a crescentic glomerulonephritis with medullary lymphomonocytic infiltration. In the biopsy specimen, only five glomerules were represented, and four of them were sclerotic. Anti-nuclear antibody (ANA), tested by immufluorescence with HEP-2 cells and anti-DNA, were negative; complement determination was normal. Serologies for HIV, hepatitis $B$, and hepatitis $C$ were negative. Protoplasmic anti-neutrophilic cytoplasmic antibody (p-ANCA) was positive $>1 / 80$ (RV: non-reactive) as well as myeloperoxidase (MPO) $120 \mathrm{U}(\mathrm{RV}:<20 \mathrm{U})$.

Based on the clinical and laboratory results, the patient was diagnosed with renal insufficiency, which was probably due to ANCA-related vasculitis and AS. She was referred for aortic valve surgery, after angiocoronariography ruling out coronary artery disease and normal pulmonary function test.

While waiting for the valvular surgery, she returned to the emergency room complaining of diffuse thoracic pain, dyspnea, and fatigue. She had lost $5 \mathrm{~kg}$ in 30 days. At admittance she was drowsy, cyanotic, tachypneic, tachycardic, and hypotensive, and had a room air oximetry of $76 \%$. Pulmonary examination detected diffuse bilateral rales. Plain thoracic radiography was interpreted as pulmonary congestion, and the thoracic computed tomography revealed emphysema, bronchiolectasis, diffuse ground glass opacification, and paratracheal lymphadenopathy, besides the calcification of the aortic valve and a mild pericardial effusion. Urine culture isolated Klebsiella sp. Acute pulmonary edema and urosepsis were considered as a plausible diagnosis; therefore, the patient was treated with antibiotics, vasoactive intravenous drugs, respiratory assistance, and a 3-day course of methylprednisolone pulse therapy because of the suspicion of a pulmonary renal syndrome. After the corticosteroid, the respiratory function improved, but it could not be ascertained whether a cause and effect correlation was related to the corticosteroid?

The recurrent pulmonary symptoms were interpreted as a consequence of the severe AS. After clinical recovery and stabilization of the hemodynamic and pulmonary function, she was referred to a cardiology center, where she presented a new episode of acute respiratory failure, which was firstly considered to be due to acute pulmonary 
edema, but soon after admission, she had a massive hemoptysis and died.

\section{AUTOPSY FINDINGS}

At the autopsy, gross examination disclosed significant alterations in the lungs, kidneys, and heart. The lungs had extensive areas of hemorrhage (Figure 1) and both weighed $2160 \mathrm{~g}$ (RV: 700-800 g).

The kidneys showed yellowish, irregularshaped zones, suggestive of necrosis, mostly in the medullar region, but also at the cortex (Figure 2). The heart (Figure 3) weighed $560 \mathrm{~g}$ (RV: 300-350 $\mathrm{g}$ ), with left ventricular hypertrophy and moderate/ severe AS with commissural fusion. In the right atrium, a friable mass was found close to the atrioventricular groove between the coronary sinus and the atrial appendage. Additionally, there was a dark red strip in the small bowel, indicative of hemorrhagic necrosis.

At microscopy, vasculitis was detected in the pulmonary microcirculation, in capillary vessels, arterioles, and venules, with destruction of the wall causing hemorrhage (Figure 1).

Both kidneys had chronic injuries (global and segmental glomerular sclerosis with fibroblastic crescents, and tubular atrophy with interstitial fibrosis) and acute injuries (medullary angiitis) (Figure 2). Vasculitis was not found in any other organ, including the bowel (with hemorrhagic infarction) and the heart. The aortic valve showed fibrosis and calcification, without significant inflammation; and there was perivascular fibrosis. The right atrial mass showed an organized thrombus, with no signs of infection. These cardiac alterations are also displayed in Figure 3.
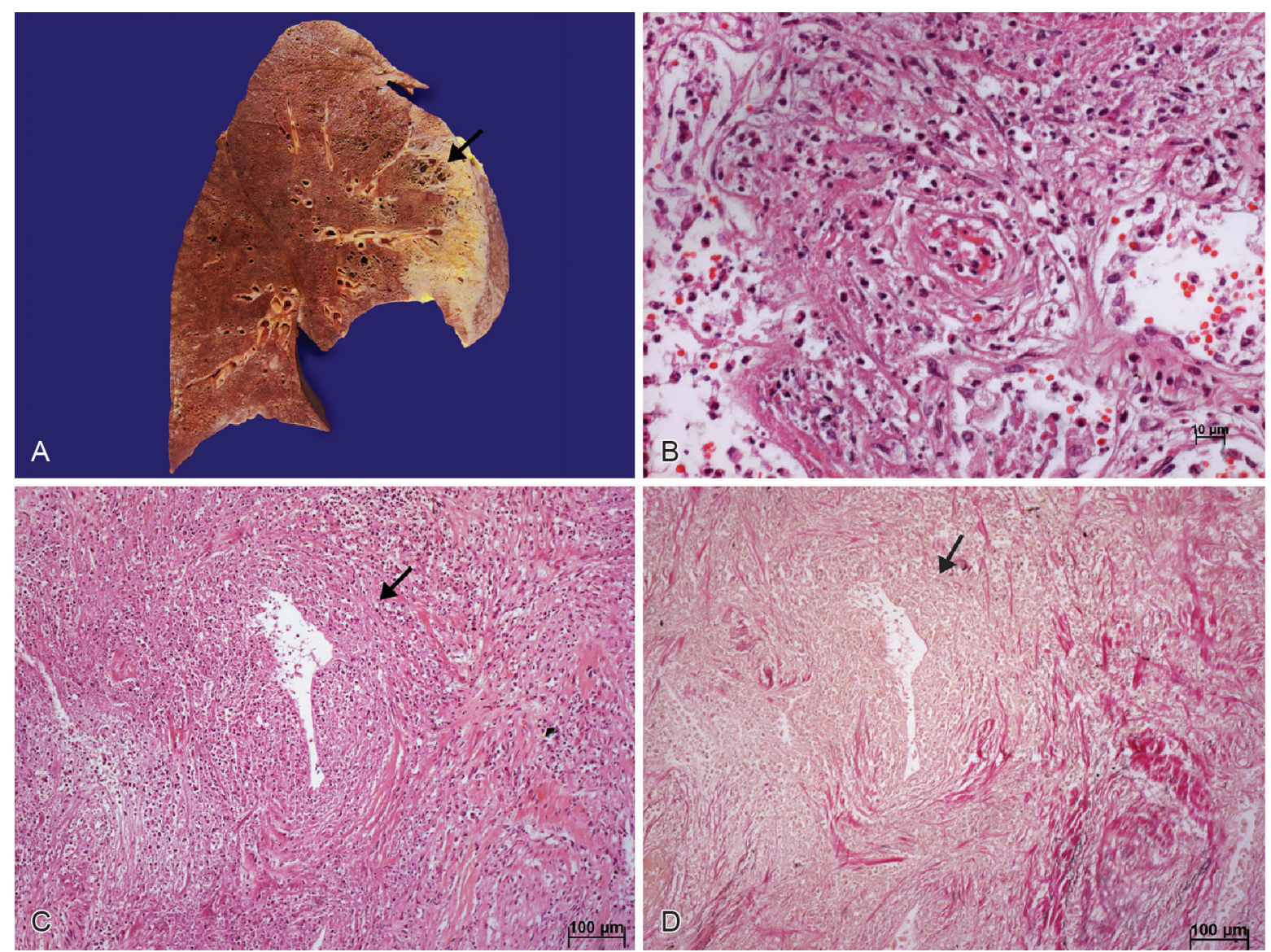

Figure 1 - A - Macroscopical view of the lungs showing diffuse hemorrhage, with a dark-reddish color, and small areas of emphysema, indicated by the orange arrow; $\mathbf{B}$ - Capillary vessel showing inflammatory cells crossing its wall (H\&E staining, objective magnification 5X); $\mathbf{C}-$ An arteriole, pointed by blue arrows, presenting inflammation, leading to $\mathbf{D}$ - destruction of the elastic laminae, which should appear stained in black. (C - H\&E staining; D- Miller's staining; objective magnification 10X). 

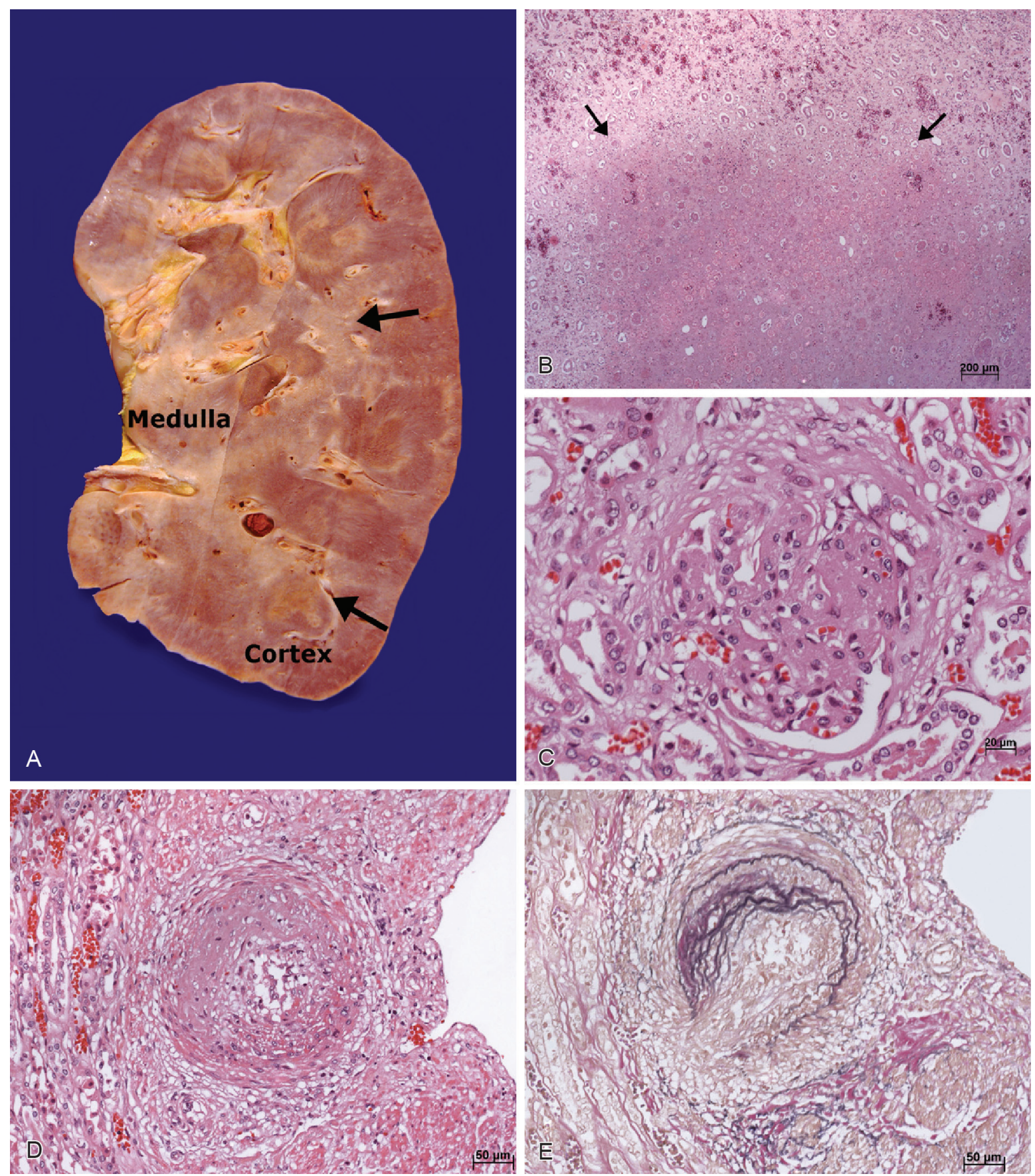

Figure 2 - Kidney medullary necrosis, indicated by arrows: A - macroscopically; and B - microscopically; C - Glomerulopathy with crescents; D - Kidney arteritis, with inflammatory cells; E - Elastic tissue (in black); destruction in the same vessel. (B, C, and D - H\&E staining; E - Miller's staining for elastic tissue. Objective magnifications: $B-5 X ; C-40 X ; D$ and $E-20 X)$.

Taking into account the positivity for p-ANCA and MPO, the main disease was considered to be microscopic polyangiitis (MPA), compromising lungs and kidneys, according to the Chapel Hill classification. ${ }^{1,2}$ Death was attributed to pulmonary hemorrhage, with hemorrhagic shock also causing a band of intestinal infarction. The right atrial thrombus was most probably caused by endocardial damage due to the dialysis catheter. Although it is a serious condition, we consider the AS to be a bystander lesion in the final event of this patient.

\section{DISCUSSION}

After the description of Dr. Adolf Kussmaul and Dr. Rudolf Maier in 1866, on what today is known as polyarteritis nodosa (PAN), all forms of vasculitis tended to be considered forms of PAN, irrespective of the size or type of vessel involved. ${ }^{3}$ Since then, other investigators have described other forms of vasculitis according to clinical and pathological particularities. In 1985, Savage et al. ${ }^{4}$ suggested that MPA was a distinct entity characterized by a 

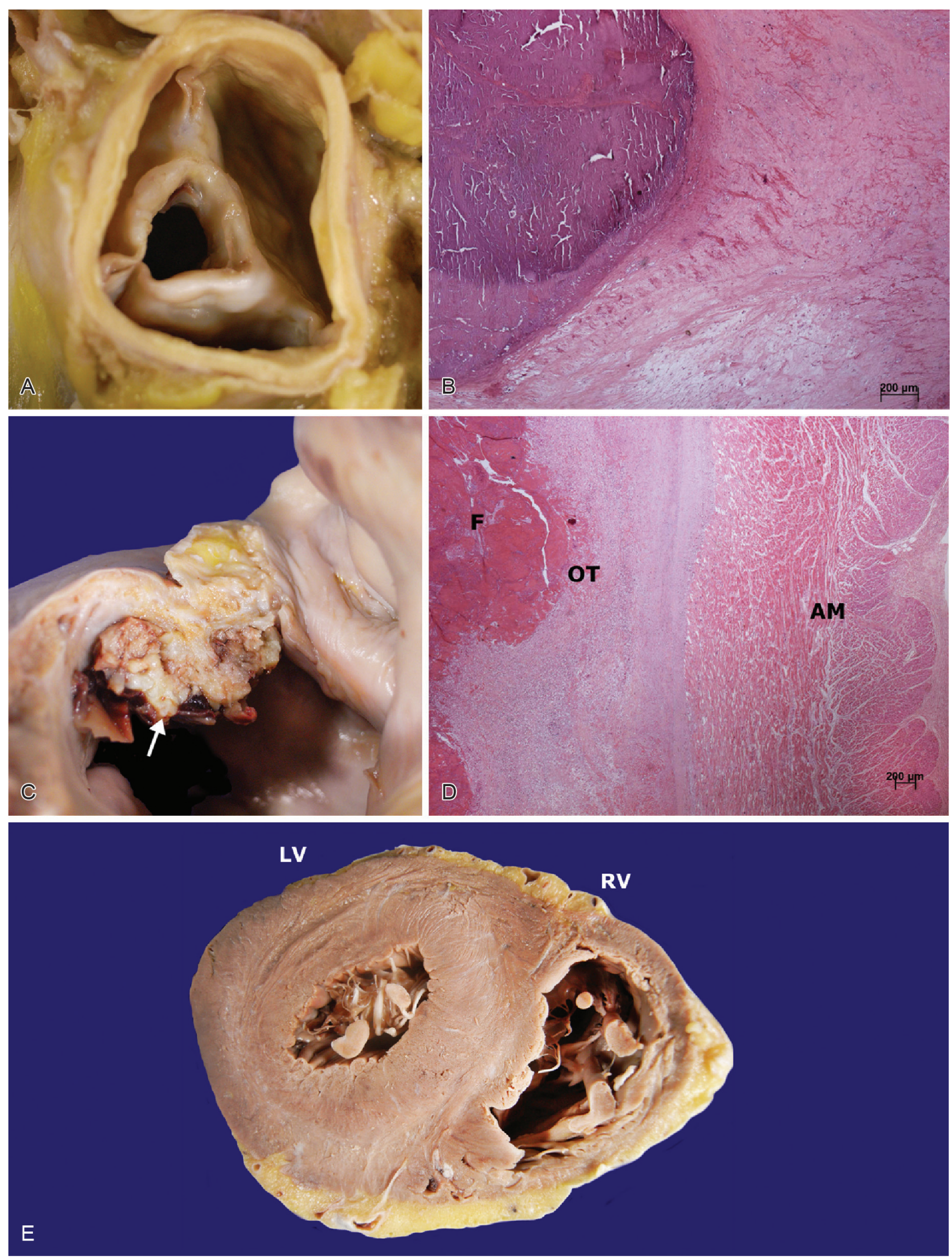

Figure 3 - Heart alterations. A - Aortic valve stenosis. There is commissural fusion and diffuse thickening of the semilunar leaflets; $\mathbf{B}$ - A microscopic aspect of the aortic valve, with dense calcification (in purple) and no inflammatory cells (H\&E, objective magnification 5X); C - Mass (thrombus), indicated by the arrow, attached to the right atrial wall; $\mathbf{D}-$ Microscopic view of the mass showed in $\mathrm{C}$, constituted by organizing thrombus (H\&E, objective magnification 2.5X); E - Short-axis section of the heart, showing left ventricular hypertrophy; $\mathrm{AM}=$ right atrial myocardium $\mathrm{F}=$ fibrin; $\mathrm{LV}=$ left ventricle; $\mathrm{OT}=$ organizing portion of the thrombus; $\mathrm{RV}=$ right ventricle. 
rapidly progressive glomerulonephritis frequently in conjunction with pulmonary capillaritis, associatedin the vast majority of cases-with the presence of ANCA.

MPA is a small vessels ANCA-associated vasculitis. In these cases, ANCA is predominantly directed against myeloperoxidase (MPO-ANCA) or proteinase 3 (PR3-ANCA). ${ }^{5}$

The incidence of MPA is not well established since it varies depending on geographical differences. In the United Kingdom, the incidence of MPA was shown to be $5.9 /$ million, during the period 1988-2010. ${ }^{6} \mathrm{~A}$ slight predominance among males is observed and the age of presentation peaks around 60-65 years of age. ${ }^{5}$ Although of unclear role, silica exposure has been suggested as a possible environmental factor linked with MPA etiopathogenesis. ${ }^{7}$ However, strong evidence argues favorably for an autoimmune mechanism as the main etiology of MPA. ${ }^{5}$ In this setting, the cornerstone is the presence of ANCA. Of patients with MPA, $95 \%$ are ANCA positive and $70 \%$ of them are MPO-ANCA, the titer of which frequently rises preceding the disease activity. ${ }^{8}$ The kidneys are affected in $80-90 \%$ of cases of MPA, and sometimes no other organs are affected. Clinically, renal involvement of MPA presents as a slight hematuria with non-massive proteinuria accompanied by a rapid deterioration of renal function. ${ }^{9}$ Lung involvement, which is somewhat less frequent $(30 \%$ of the cases), ${ }^{10,11}$ clinically presents as dyspnea, cough, and hemoptysis - the latter being associated with poor prognosis. Pulmonary imaging consists of an alveolar filling pattern, 5,12 which challenges the differential diagnosis due to its unspecificity. Weight loss, fever, arthralgia, myalgia, purpura, and peripheral nervous system involvement may also be present, but this occurs less frequently. ${ }^{5}$

The original Chapel Hill Consensus Conference Nomenclature of Vasculitides, ${ }^{1}$ revised in $2012,{ }^{2}$ defines MAP as a small vessel (arterioles, capillaries, venules) pauci-immune necrotizing vasculitis, although medium-sized arteries may be present. $^{5}$ Involvement of the skin resembles leukocytoclastic vasculitis; capillaritis with fibrinoid necrosis is the typical finding in the lungs, while necrotizing crescentic glomerulonephritis characterizes the renal lesions. Focal thrombosis of glomerular capillaries with fibrinoid necrosis is an early lesion, which is followed by rupture of the glomerular basement membrane and extracapillary proliferation. This results in crescent formation, which initially appear as cellular crescents, but later on become more fibrous crescents. ${ }^{5}$

The patient presented herein was admitted with the diagnosis of probable acute renal failure, initially interpreted as caused by the NSAID recently used, iron-deficiency anemia and an AS, which was asymptomatic for the last 11 years. The renal function did not show any improvement with corticosteroids, the presumed offending drug was withdrawn, and ultrasound found apparently normal kidneys. Therefore, a renal biopsy was undertaken, but the histology was not conclusive, due to the exiguity of the sample. Nevertheless, urinalysis and the presence of ANCA pointed towards a probable autoimmune etiology for the renal function impairment.

Although gastrointestinal involvement in patients with granulomatosis with polyangiitis (GPA) or MPA is rare, ${ }^{13}$ and at the autopsy of this case no vasculitis was found at the infarcted bowel segment, it would not be absurd to suspect gastrointestinal involvement since in the patient's initial symptom was abdominal pain. Moreover, there was an iron deficiency anemia, and at the autopsy a hemorrhagic infarction band was found. We preferred to consider this finding as result of the hemodynamic shock (because of the lack of histological evidence of vasculitis), but we are not totally convinced because in the case of infarction due to arterial hypotension we should expect larger ischemic areas.

The presence of the AS with a peak left ventricular/aortic pressure gradient of $92 \mathrm{mmHg}$ somehow overshadowed the clinical reasoning, directing it towards the hemodynamic effects of the AS to explain the respiratory symptoms. Unfortunately, this pressure gradient was not measured during the cardiac catheterization, and therefore could not be compared with the echo Doppler measurement. Even with the absence of the mean pressure gradient, valvular area information, and flow velocity, the AS was considered severe. ${ }^{14}$ The respiratory features of this patient were probably first misinterpreted when she was diagnosed with pneumonia (the first episode preceding the hospital admission), and acute pulmonary edema (during further events) due to hemodynamic effects caused by the AS. Pulmonary vasculitis was not considered as the cause of the respiratory discomfort. Therefore, the treatment of the patient was focused on the aortic valve replacement. However, the suspicion of ANCA-related vasculitis was considered at one point, so methyl prednisolone pulse therapy was 
prescribed. Unfortunately, the immunosuppression did not go further due to the presence of infection and the lack of support on the hypothesis of pulmonary vasculitis.

The process of reaching a diagnosis is complex, involving recognition of signs and symptoms, clinical reasoning and experience, and, whenever possible, the demonstration of the pathologic process or any laboratory surrogate finding. ${ }^{15}$ In this case report, the diagnosis of MPA was left in the background, even in the presence of such evidence, illustrating how clinical reasoning can be diverted when some data (cardiac murmur and echodopplercardiogram) are more valued than others. With the findings of the autopsy, we surprisingly concluded that the medical staff may have overestimated the role of the AS concerning the respiratory features. It is not unlikely that AS was merely a bystander at this time.

It is likely that the higher incidence of AS compared with MPA, and the medical staff's familiarity to it, led to the treatment of AS being prioritized.

Although committing some medium-sized vessels, what is unusual but may still happen, ${ }^{15}$ the vasculitis of this patient was classified as MPA. Clinical features (including the lack of eosinophilia and history of asthma), the positivity for ANCA antibodies, and the pathologic findings involving small vessels of the kidneys and lungs without granulomas, support this diagnosis.

The cause of the aortic valve stenosis was not well disclosed. Aortic lesions-mostly aortic regurgitation-have been described in a few patients with vasculitis ${ }^{16}$ due to aortitis, which was absent in our case. The autopsy showed that the patient had no infective endocarditis, which may be either associated with or simulate the clinical features of vasculitides. ${ }^{17,18}$ Thus we considered the valve disease as a possible consequence of previous rheumatic heart disease, taking into account the commissural fusion and epidemiological data, even though there was no history of joint pain in the childhood, no significant inflammation at necropsy, and no concomitant mitral involvement. (Although decreasing, rheumatic valve disease is relatively common in Brazil). Another possibility to be considered is senile calcification, but such a condition is unusual under 70 years of age.
We would like to remind the unicist clinical reasoning/thinking, which emphasizes, whenever possible, the use a sole entity to explain all signs and symptoms. We do not discard the doctors' belief that an aortic valve replacement was necessary, but faced with the autopsy findings, we conclude that it should have been postponed until the autoimmune disease was under control.

\section{REFERENCES}

1. Jennette JC, Falk RJ, Andrassy K, et al. Nomenclature of systemic vasculitis. Proposal of an international consensus conference. Arthritis Rheum. 1994;37:187-92. http://dx.doi. org/10.1002/art.37715

2. Jennette JC, Falk RJ, Bacon PA, et al. 2012 Revised international Chapel Hill consensus conference nomenclature of vasculitides. Arthritis Rheum. 2013;65:1-11. http://dx.doi. org/10.1002/art.37715

3. Campos FPF, Geller SA. Churg-Strauss Syndrome: a syndrome described on clinical observation and autopsy findings [editorial]. Autopsy Case Rep [Internet]. 2013;3:1-4.

4. Savage C, Winearts C, Evans D, Rees A, Lockwood C. Microscopic polyarteritis: presentation, pathology and prognosis. Q J Med. 1985;56:467-83.

5. Kallenberg CGM. The diagnosis and classification of microscopic polyangiitis. J Autoimmun. 2014 Jan 22. In Press. http://dx.doi.org/10.1016/j.jaut.2014.01.023

6. Watts RA, Mooney J, Skinner J, Scott DJ, Macgreggor AJ. The contrasting epidemiology of granulomatosis with polyangiitis and microscopic polyangiitis. Rheumatology. 2012;51:92631. http://dx.doi.org/10.1093/rheumatology/ker454

7. Hogan SL, Satterly KK, Dooley MA, Nachman PH, Jennette JC, Falk RJ. Silica exposure in antineutrophil cytoplasmic autoantibody-associated glomerulonephritis and lupus nephritis. J Am Soc Nephrol. 2001;12:134-42.

8. Terrier B, Saadoun D, Sane D, et al. Antimyeloperoxidase antibodies are a useful marker of disease activity in antineutrophil cytoplasmic antibody-associated vasculitides. Ann Rheum Dis. 2009;68:1564-71. http://dx.doi.org/10.1136/ ard.2008.094714

9. Guillevin L, Durand-Gasselin B, Cevallos R, et al. Microscopic polyangiitis: clinical and laboratory findings in eighty-five patients. Arthritis Rheum. 1999;42:421-30. http://dx.doi. org/10.1002/1529-0131(199904)42:3\%3C421::AIDANR5\%3E3.0.CO;2-6

10. Akikusa B, Sato T, Ogawa M, Ueda S, Kondo Y. Necrotizing alveolar capillaritis in autopsy cases of microscopic polyangiitis: incidence, histopathogenesis and relationship with systemic vasculitis. Arch Pathol Lab Med. 1997;121:144-9. 
11. Brown KK. Pulmonary vasculitis. Pulmonary Vasculitis. Proc Am Thorac Soc. 2006;3:48-57. http://dx.doi.org/10.1513/ pats.200511-120JH

12. Niles JL, Bottinger EP, Saurina GR, et al. The syndrome of lung hemorrhage and nephritis is usually an ANCA-associated condition. Arch Intern Med. 1996;156:440-5. http://dx.doi. org/10.1001/archinte.1996.00440040118013

13. Latus J, Koetter I, Fritz P, et al. Gastrointestinal involvement in granulomatosis with polyangiitis and microscopic polyangiitis: histological features and outcome. Int J Rheum Dis. 2013 Oct 29. http://dx.doi.org/10.1111/1756-185X.12203

14. Vahanian A, Otto CM. Risk stratification of patients with aortic stenosis. Eur Heart J. 2010;31:416-23. http://dx.doi. org/10.1093/eurheartj/ehp575.

\section{Conflict of interest: None}

Submitted on: $12^{\text {th }}$ February 2014

Accepted on: 20 $0^{\text {th }}$ March 2014

Correspondence: Instituto do Coração

HCFMUSP

Av. Dr. Enéas Carvalho de Aguiar, 44 - São Paulo/SP - Brazil

CEP: 05403-000 - Phone: +55 (11) 2661-5251

E-mail: paulo.gutierrez@incor.usp.br
15. Jennette JC, Falk RJ. The role of pathology in the diagnosis of systemic vasculitis. Clin Exp Rheumatol. 2007; 25:S52-6.

16. Lacoste C, Mansencal N, Ben M'rad M, et al. Valvular involvement in ANCA-associated systemic vasculitis: a case report and literature review. BMC Musculoskelet Disord. 2011;12:50. http://dx.doi.org/10.1186/1471-2474-12-50

17. Chirinos JA, Corrales-Medina VF, Garcia S, Lichtstein DM, Bisno AL, Chakko S. Endocarditis associated with antineutrophil cytoplasmic antibodies: a case report and review of the literature. Clin Rheumatol. 2007;26:590-5. http://dx.doi.org/10.1007/s10067-005-0176-z

18. Teoh LS, Hart HH, Soh MC, et al. Bartonella henselae aortic valve endocarditis mimicking systemic vasculitis. BMJ Case Rep. 2010. pii: bcr0420102945. http://dx.doi.org/10.1136/ bcr.04.2010.2945 